\title{
Segmentación de padres acorde a estrategias educativas familiares en hogares con hijos entre primero y cuarto año de educación básica de la ciudad de Guayaquil
}

\section{Parent segmentation according to family educational strategies in homes with children between first and fourth year of basic education of the city of Guayaquil}

Jorge Izaguirre Olmedo

Universidad Internacional del Ecuador, Ecuador

Viviana Medina Vergara

Universidad Internacional del Ecuador, Ecuador

Autor por correspondencia: joizaguirreol@uide.edu.ec, vimedinave@uide.edu.ec

Fecha de recepción: 21 de Febrero de 2018 - Fecha de aceptación: 15 de agosto de 2018

\section{Resumen}

La presente investigación consiste en un análisis de conglomerados de una muestra de 400 hogares con hijos entre primero y cuarto año de educación básica en la ciudad de Guayaquil. El objetivo es agrupar a los padres o apoderados en diferentes segmentos que cumplan con características similares entre sí y distintas entre otros grupos. Para el procesamiento de datos se utilizó el paquete estadístico SPSS y la herramienta de Análisis de Conglomerados por el método jerárquico. Como principal resultado se muestran tres segmentos con sus respectivas características socioeconómicas y de Estrategias Educativas Familiares (EEF).

Palabras Claves: prácticas educativas parentales; análisis de conglomerados; varianza

\begin{abstract}
This research refers to a cluster analysis of a sample of 400 families with children between first and fourth grade of basic education in Guayaquil. The purpose is to join the parents in different groups that has similar characteristics between them and different with others. To process the data, the author used the statistic program SPSS and the Analysis of Clusters tool. As a main result, there are three different groups that differ in economic characteristics and Educational Practices of Parents.
\end{abstract}

Key words: educational practices of parents; clusters analysis; variance 


\section{Introducción}

Bourdieu \& Wacquant (1993/1995) definen a las Estrategias Educativas Familiares (EEF) como el despliegue de líneas de acción coherentes que obedecen a un patrón específico, sin que sigan reglas conscientes determinadas por un estratega. Así mismo, Bourdieu (2011), citado por Gubbins \& Ibarra (2016), señala que las EEF son "la expresión de un complejo sistema de percepciones, valoraciones, preferencias, decisiones y disposiciones que responden, y a su vez estructuran mentalidades y estilos de vida específicas en familias de distintos GSE” (pág 4).

Por su parte, Gubbins \& Ibarra (2016) indican que las EEF son aquellas estrategias que eligen las familias por iniciativa propia, acorde a sus aspiraciones y conocimientos, para ayudar a sus hijos en la educación. En este sentido, señalan los autores, se pueden identificar cuatro dimensiones en las EEF: capital socio-cultural, Disposición Parental hacia la Escuela, Aspiraciones Parentales y Prácticas Educativas Parentales.

Acorde a Weiss, Bouffard, Bridgall y Edmund (2009), uno de los factores que tiene mayor influencia en el logro académico, es el involucramiento familiar. Esta idea fue resaltada por Epstein (2013) al probar que existe una corresponsabilidad de la escuela, la familia y la comunidad en la formulación de estrategias que impulsen el desarrollo cognitivo del niño. Posteriormente, López \& Caspe (2014), indicaron que el involucramiento familiar es parte de la responsabilidad compartida de la escuela y la familia y que debe mantenerse hasta la adultez temprana.

El Ministerio de Educación del Ecuador (2015) señala la importancia que tienen las relaciones de las escuelas con las familias en el contexto de aprendizaje. En este sentido, es necesario estudiar las EEF y el respectivo involucramiento familiar de tal forma que se cuente con información suficiente para abordar esta problemática.

Las investigaciones realizadas sobre EEF en el Ecuador se han basado en la descripción de dichas estrategias (Merizalde, 2017; Ycaza, 2017) y en las correlaciones que existen entre ellas (Izaguirre \& Medina, 2017). Partiendo de este punto, la presente investigación tiene por objeto poder identificar grupos o segmentos de hogares que compartan características específicas en EEF. Una vez identificados y caracterizados dichos segmentos, se podrán determinar mecanismos de intervención específicos para cada grupo de familias que permitan la mejora de sus estrategias orientadas al apoyo educativo de los hijos.

Este estudio es de tipo exploratorio puesto que la técnica de análisis de conglomerados no permite realizar inferencia estadística (De-La-Fuente, 2011).

\section{Metodología}

Esta investigación es de tipo cuantitativa, descriptiva, no inferencial. Para la obtención de los datos se utilizaron 400 encuestas realizadas a padres o madres de familia de niños que estudian del primero al cuarto año de educación básica, 100 encuestas de primer año, 100 del segundo, 100 del tercero y 100 del cuarto año. El cuestionario utilizado corresponde al propuesto por Gubbins \& Ibarra (2016) con la debida contextualización para el Ecuador. Las encuestas fueron autoadministradas y se desarrollaron dentro de las instituciones educativas, con la debida 
autorización de los directores de los planteles. Para el tratamiento de los datos se utilizó el paquete estadístico SPSS.

Las variables recogidas, que en su mayoría son de carácter ordinal y se levantaron a través de escalas de Likert de 4 niveles, pueden clasificarse en cinco grupos: caracterización del estudiante, el apoderado y la familia; capital socio-cultural de la familia; disposición parental; aspiraciones parentales y prácticas educativas. Estos grupos son los que se utilizaron para la obtención de los segmentos.

El Análisis de Conglomerados, es una técnica estadística multivariante que tiene por objeto la agrupación de variables o individuos a través de minimización de varianza dentro del grupo y la maximización de la misma con otros grupos. Es una técnica descriptiva, ateórica y no inferencial, puesto que no tiene bases estadísticas sobre las que deducir información para una población a partir de una muestra. Se define como una técnica exploratoria, descriptiva pero no explicativa (De-La-Fuente, 2011).

Para la estimación del análisis de conglomerados, se utilizó la herramienta de conglomerados jerárquicos. El método empleado fue vinculación inter-grupos y la medida empleada fue chi-cuadrado debido a que las variables utilizadas no eran cuantitativas.

\section{Revisión de la literatura}

Las EEF son todas aquellas características, prácticas y aspiraciones que tienen los hogares con respecto a la educación de sus hijos (Bourdieu \& Passeron, 1977; Contreras, Figueroa, \& Espina, 2012; Epstein, 2013; Gubbins, 2014; López \& Caspe, 2014). Gubbins \& Ibarra (2016) señalan la existencia de cuatro dimensiones dentro de estas estrategias: el capital económico-cultural de los padres; la disposición parental hacia la escuela; las aspiraciones parentales; y las prácticas educativas parentales.

Acorde a Weiss, Bouffard, Bridgall \& Edmund (2009), el capital económico - cultural puede generar brechas en el desarrollo cognitivo del niño y en las estrategias educativas de los padres. Ycaza (2017) desarrolló un estudio en Guayaquil utilizando el cuestionario desarrollado por Gubbins \& Ibarra (2016) para obtener información y relacionar el ingreso del apoderado con algunos componentes de las prácticas educativas como incentivo a la lectura, exigencia de lugares específicos para la realización de tareas y discusión con los hijos sobre temas de actualidad. Concluyó que existen diferencias estadísticas en las prácticas educativas ante distintos niveles de ingreso. Para testear la diferencia estadística aplicó pruebas chi cuadrado.

Con respecto a la disposición parental hacia la escuela, se conoce que los hogares que se involucran más en la institución, consiguen que sus hijos obtengan mejores logros académicos (Rivera \& Milicic, 2006; Sallés \& Ger, 2011). Así mismo, los hogares que muestran una mejor disposición hacia la escuela, presentan mayor cantidad de prácticas educativas (Izaguirre \& Medina, 2017).

En referencia a las aspiraciones parentales, Jeynes W. (2003) y Catsambis (2001), ambos citados por Weiss et al. (2009); señalaron que existe una relación positiva entre dichas 
aspiraciones o expectativas y el logro académico de los hijos. Izaguirre \& Medina (2017) mostraron que las aspiraciones tienen relación positiva y significativa con el nivel de prácticas educativas desarrolladas por los apoderados.

Las prácticas educativas son las acciones concretas de los apoderados o padres que se orientan al éxito académico de los hijos (Darling \& Steinberg, 1993; citados por Rivera \& Milicic, 2006). El nivel o cantidad de estas prácticas están correlacionadas positivamente con el nivel de educación del apoderado y su frecuencia de lectura, el nivel de integración del hogar, la disposición parental hacia la escuela y las prácticas parentales; así como está correlacionado negativamente con el nivel de ingreso del apoderado (Izaguirre \& Medina, 2017). Acorde a Baumrind (1971), citado por Weiss et al. (2009), se identifican tres estilos de prácticas parentales: autoritativos, permisivos y autoritarios.

\section{Resultados}

Como principal resultado se pudo clasificar la muestra en tres conglomerados, dos de ellos con una fuerte representación, vinculada fuertemente con la edad; y un último clúster más pequeño, pero con características claramente diferenciadas.

La Tabla 1 muestra la distribución de frecuencia de los clúster. El primer grupo, denominado a partir de ahora como Metanoiers, comprende un 63\% de la muestra, constituyendo el clúster más grande. El segundo grupo será denominado como Modernos y representa el 28.5\%; finalmente, el tercer grupo, a los que se les llamarán Tradicionalistas, cuenta con 5\% de los individuos de la muestra.

Tabla 1. Distribución de Frecuencias de los Conglomerados

\begin{tabular}{llrrrr}
\hline & Frecuencia & Porcentaje & $\begin{array}{c}\text { Porcentaje } \\
\text { válido }\end{array}$ & $\begin{array}{c}\text { Porcentaje } \\
\text { acumulado }\end{array}$ \\
\hline Válidos & Metanoiers & 252 & 63,0 & 65,5 & 65,5 \\
& Modernos & 114 & 28,5 & 29,6 & 95,1 \\
& Tradicionalistas & 19 & 4,8 & 4,9 & 100,0 \\
& Total & 385 & 96,3 & 100,0 & \\
Perdidos & Sistema & 15 & 3,8 & & \\
Total & & 400 & 100,0 & & \\
\hline
\end{tabular}

Para una mejor presentación de los resultados obtenidos, las cuatro dimensiones de las estrategias educativas familiares serán presentadas en tablas independientes con su respectivo análisis. En este sentido, la Tabla 2 muestra las características del capital económico - cultural de los padres encuestados y clasificados en cada segmento.

Tabla 2. Capital Económico - Cultural de los Padres 


\begin{tabular}{|c|c|c|c|}
\hline Variables & Metanoiers & Modernos & Tradicionalistas \\
\hline $\begin{array}{l}\text { Madre como } \\
\text { apoderada }\end{array}$ & $68 \%$ & $89 \%$ & $68 \%$ \\
\hline Edad & $\begin{array}{c}65 \% \text { entre } 30 \text { y } 40 \\
\text { años }\end{array}$ & $47 \%$ entre 25 y 35 años & $47 \%$ más de 40 años \\
\hline Estudios & $\begin{array}{c}72 \% \text { tiene } \\
\text { universidad completa o } \\
\text { superior }\end{array}$ & $\begin{array}{l}75 \% \text { está entre educación } \\
\text { básica completa y media } \\
\text { completa }\end{array}$ & $\begin{array}{c}82 \% \text { tiene educación } \\
\text { básica completa o inferior }\end{array}$ \\
\hline $\begin{array}{l}\text { Actividad } \\
\text { principal }\end{array}$ & $\begin{array}{l}83 \% \text { tiene trabajo o } \\
\text { negocio permanente }\end{array}$ & $\begin{array}{l}68 \% \text { quehaceres del hogar } \\
\text { y trabajos eventuales }\end{array}$ & $\begin{array}{c}69 \% \text { quehaceres del } \\
\text { hogar y trabajos eventuales }\end{array}$ \\
\hline $\begin{array}{l}\text { Hijos menores } 18 \\
\text { años }\end{array}$ & $\begin{array}{c}82 \% \text { tienen } 1 \text { o } 2 \\
\text { hijos menores de edad }\end{array}$ & $\begin{array}{l}52 \% \text { entre } 2 \text { y } 3 \text { hijos } \\
\text { menores de edad }\end{array}$ & $\begin{array}{l}61 \% \text { tienen } 1 \text { o } 2 \text { hijos } \\
\text { menores de edad }\end{array}$ \\
\hline Ingreso promedio & $\begin{array}{l}72 \% \$ 1,500 \text { o } \\
\text { superior }\end{array}$ & $82 \%$ menos de $\$ 470$ & $68 \%$ menos de $\$ 366$ \\
\hline Lectura de libros & $\begin{array}{l}\text { 14\% todos los días } \\
22 \% 1 \text { vez por semana } \\
17 \% \text { nunca }\end{array}$ & $\begin{array}{c}30 \% \text { todos los días } \\
37 \% 1 \text { vez por semana } \\
6 \% \text { nunca }\end{array}$ & $\begin{array}{c}21 \% \text { todos los días } \\
53 \% 1 \text { vez por semana } \\
26 \% \text { nunca }\end{array}$ \\
\hline $\begin{array}{l}\text { Practicar juegos } \\
\text { didácticos }\end{array}$ & $\begin{array}{l}69 \% \text { entre casi nunca } \\
\text { y ocasionalmente }\end{array}$ & $\begin{array}{l}72 \% \text { entre ocasionalmente } \\
\text { y muy frecuentemente }\end{array}$ & $59 \%$ nunca \\
\hline $\begin{array}{l}\text { Resolver tareas y } \\
\text { dudas del } \\
\text { estudiante }\end{array}$ & $\begin{array}{c}21 \% \text { familiares } \\
36 \% \text { internet } \\
17 \% \text { lo resuelve solo }\end{array}$ & $\begin{array}{c}46 \% \text { familiares } \\
12 \% \text { internet } \\
25 \% \text { lo resuelve solo }\end{array}$ & $\begin{array}{c}44 \% \text { familiares } \\
11 \% \text { internet } \\
28 \% \text { lo resuelve solo }\end{array}$ \\
\hline
\end{tabular}

Como primer dato, en cuanto a la estructura familiar se refiere, en los tres segmentos el mayor porcentaje de los apoderados corresponde a las madres, no obstante, en el segmento de Modernos, el porcentaje alcanza el 89\%. Por otro lado, 4 de cada 5 Metanoiers, así como el $61 \%$ de los Tradicionales, tienen de 1 a 2 hijos menores de 18 años; mientras que el 52\% de los Modernos tienen de 2 a 3 hijos en ese rango de edad.

En lo que respecta a la edad de los apoderados, los Metanoiers están comprendidos, en su mayoría, por padres entre los 30 y los 40 años de edad; mientras que los Modernos se encuentran, aproximadamente en un 50\%, entre los 25 y los 35. Por último, los Tradicionalistas están compuestos, en un $47 \%$, por padres de más de 40 años.

El nivel de estudios es una de las principales diferencias de los segmentos. El $72 \%$ de los Metanoiers tienen educación universitaria completa o superior. El 75\% de los Modernos tienen educación desde básica completa hasta media completa. En contraste, los Tradicionalistas tienen, en un $82 \%$, educación básica completa o inferior.

En lo que corresponde a actividad principal realizada por el apoderado, los segmentos Modernos y Tradicionalistas son similares al reportar en un 68\% y 69\%, respectivamente, que se dedican a labores domésticas y/o trabajos eventuales. Por su parte, los Metanoiers, en un $82 \%$, reportan tener un trabajo o negocio permanente. Esta información se relaciona con el nivel de 
ingresos reportado. Los Metanoiers, en un 72\%, reportan ingresos mensuales de $\$ 1,500$ o superior. Los Modernos (82\%) reportan un ingreso menor a $\$ 470$, mientras que los Tradicionalistas (68\%) reportan ganar menos de $\$ 366$.

En cuanto al capital cultural de los padres, se consultó sobre la frecuencia de lectura, la dinámica de juegos didácticos y el apoyo en la resolución de tareas a los hijos. En lo referente a la lectura, el 14\% de los Metanoiers leen diariamente, mientras que los Modernos lo hacen en un $30 \%$ y los Tradicionales en un $21 \%$. En contraste, para el primer grupo, el $17 \%$ nunca lee, frente al $6 \%$ del segundo grupo y al $26 \%$ del tercero. Se observa, en resumen, que el grupo de Modernos tienen mayor tendencia a la lectura que los otros dos grupos consultados.

Por otro lado, en lo que respecta a los juegos didácticos, las diferencias son marcadas. Los Metanoiers, en un 69\%, casi nunca los practican o lo hacen ocasionalmente. Los Modernos, en contraste, practican estos juegos entre ocasionalmente y muy frecuentemente en un $72 \%$ de los encuestados. Por último, los Tradicionales, en su mayoría con el 59\%, nunca practican estos juegos.

Finalmente, en la categoría de ayuda en tareas y dudas del estudiante, los Metanoiers respondieron que consultan en internet en un 36\%, los Modernos y los Tradicionales acuden a familiares en un $44 \%$ y $46 \%$, respectivamente. De esa misma pregunta se obtuvo que el $17 \%$ de los hijos de Metanoiers resuelven solos sus tareas y dudas, mientras que para los Modernos y Tradicionales los porcentajes alcanzan el 25 y $28 \%$, respectivamente; representando un mayor nivel de independencia en sus estudios.

Tabla 3. Disposición Parental hacia la Escuela

\begin{tabular}{|c|c|c|c|}
\hline Variables & Metanoiers & Modernos & Tradicionales \\
\hline $\begin{array}{l}\text { Asiste a reuniones de } \\
\text { apoderados }\end{array}$ & $63 \%$ casi siempre & $76 \%$ siempre & $63 \%$ siempre \\
\hline $\begin{array}{c}\text { Voluntariado en } \\
\text { actividades dentro de la } \\
\text { escuela }\end{array}$ & $\begin{array}{l}50 \% \text { entre } \\
\text { ocasionalemente y muy } \\
\text { frecuentemente }\end{array}$ & $\begin{array}{l}66 \% \text { entre } \\
\text { nunca y casi } \\
\text { nunca }\end{array}$ & $67 \%$ nunca \\
\hline $\begin{array}{l}\text { Acompañar al profesor en } \\
\text { actividades fuera de la } \\
\text { escuela }\end{array}$ & No hay tendencia & $51 \%$ nunca & $73 \%$ nunca \\
\hline $\begin{array}{l}\text { Razón de falta de } \\
\text { involucramiento }\end{array}$ & $77 \%$ por trabajo & $\begin{array}{c}43 \% \text { por } \\
\text { trabajo } \\
47 \% \text { por tareas } \\
\text { del hogar }\end{array}$ & $\begin{array}{l}45 \% \text { por trabajo } \\
55 \% \text { por tareas del } \\
\text { hogar }\end{array}$ \\
\hline
\end{tabular}

La Tabla 3 muestra los resultados y diferencias de los grupos con respecto a la disposición parental hacia la escuela. Como primer punto se puede observar un mayor nivel de asistencia a reuniones de apoderados por parte de los Modernos. Este grupo reporta, en un 76\%, que siempre asisten a las reuniones. Con respecto a los Metanoiers, la mayor concentración de 
padres se ubicó en el nivel de casi siempre con un 63\%. Mientras que los Tradicionales, con el mismo $63 \%$, reportaron asistir siempre a dichas reuniones.

En lo que respecta al voluntariado en actividades dentro de la escuela, la mayor concentración de los Metanoiers se ubicó entre ocasionalmente y muy frecuentemente con un $50 \%$. En el caso de los Modernos, el $66 \%$ se ubicó entre nunca y casi nunca; mientras que los Tradicionales en un $67 \%$ respondieron que nunca asisten al voluntariado. Este resultado denota una mayor tendencia de compromiso con actividades voluntarias para el primer grupo.

Una de las consultas en cuanto a la disposición parental fue el acompañar al profesor en actividades que se realizan fuera de la escuela. Al respecto, no se encontró una clara tendencia para los Metanoiers. Por su parte, los Modernos y los Tradicionales indicaron que nunca en un $51 \%$ y $73 \%$, respectivamente.

Finalmente, se consultó a los padres el porqué de la falta de involucramiento con la escuela en las actividades previamente mencionadas. Los Metanoiers, en un 77\%, respondieron que era por trabajo. Los Modernos indicaron que era por trabajos del hogar en un $47 \%$ y por trabajo en un $43 \%$. Finalmente, los Tradicionales reportaron, en un 55\%, que era por tareas del hogar y en un $45 \%$ que era por razones laborales.

Tabla 4. Prácticas Educativas Parentales

\begin{tabular}{|c|c|c|c|}
\hline Variables & Metanoiers & Modernos & Tradicionales \\
\hline Exige horario para tareas & $86 \%$ & $87 \%$ & $68 \%$ \\
\hline $\begin{array}{c}\text { Establece horarios para tv } \\
\text { y juegos }\end{array}$ & $81 \%$ & $76 \%$ & $53 \%$ \\
\hline $\begin{array}{l}\text { Establece horarios para } \\
\text { uso de computadoras }\end{array}$ & $78 \%$ & $59 \%$ & $11 \%$ \\
\hline Impone hora de acostarse & $84 \%$ & $85 \%$ & $53 \%$ \\
\hline $\begin{array}{l}\text { Exige realizar tareas en } \\
\text { lugar específico }\end{array}$ & $56 \%$ & $87 \%$ & $37 \%$ \\
\hline Leerle libros & $\begin{array}{l}73 \% \text { ocasionalmente o } \\
\text { muy frecuentemente }\end{array}$ & $\begin{array}{l}81 \% \text { ocasionalmente o } \\
\text { muy frecuentemente }\end{array}$ & $\begin{array}{l}63 \% \text { nunca o casi } \\
\text { nunca }\end{array}$ \\
\hline Incentivar la lectura & $\begin{array}{l}77 \% \text { ocasionalmente o } \\
\text { muy frecuentemente }\end{array}$ & $\begin{array}{l}93 \% \text { ocasionalmente o } \\
\text { muy frecuentemente }\end{array}$ & $\begin{array}{l}74 \% \text { nunca o casi } \\
\text { nunca }\end{array}$ \\
\hline $\begin{array}{l}\text { Contarle cuentos } \\
\text { inventados }\end{array}$ & $\begin{array}{l}77 \% \text { ocasionalmente o } \\
\text { muy frecuentemente }\end{array}$ & $\begin{array}{l}67 \% \text { ocasionalmente o } \\
\text { muy frecuentemente }\end{array}$ & $89 \%$ nunca \\
\hline $\begin{array}{l}\text { Discutir con el niño sobre } \\
\text { temas de actualidad }\end{array}$ & $\begin{array}{l}81 \% \text { ocasionalmente o } \\
\text { muy frecuentemente }\end{array}$ & $\begin{array}{l}70 \% \text { ocasionalmente o } \\
\text { muy frecuentemente }\end{array}$ & $74 \%$ nunca \\
\hline
\end{tabular}




\begin{tabular}{|c|c|c|c|}
\hline $\begin{array}{c}\text { Conversar sobre la } \\
\text { importancia de la escuela }\end{array}$ & $\begin{array}{c}53 \% \text { muy } \\
\text { frecuentemente }\end{array}$ & $\begin{array}{c}81 \% \text { muy } \\
\text { frecuentemente }\end{array}$ & $\begin{array}{c}47 \% \text { nunca } \\
16 \% \\
\text { ocasionalmente }\end{array}$ \\
\hline $\begin{array}{l}\text { Revisarle las tareas } \\
\text { escolares }\end{array}$ & $48 \%$ ocasionalmente & $\begin{array}{l}86 \% \text { muy } \\
\text { frecuentemente }\end{array}$ & $47 \%$ nunca \\
\hline $\begin{array}{c}\text { Plantearle operaciones } \\
\text { matemáticas }\end{array}$ & $58 \%$ ocasionalmente & $\begin{array}{l}59 \% \text { muy } \\
\text { frecuentemente }\end{array}$ & $\begin{array}{l}42 \% \text { muy } \\
\text { frecuentemente }\end{array}$ \\
\hline $\begin{array}{l}\text { Las tareas de hoy en día } \\
\text { son más fáciles que las de } \\
\text { antes }\end{array}$ & $56 \%$ en desacuerdo & $\begin{array}{l}\text { No hay tendencia } \\
\text { específica }\end{array}$ & $62 \%$ de acuerdo \\
\hline $\begin{array}{l}\text { Dispone de tiempo para } \\
\text { ayudar en tareas }\end{array}$ & $75 \%$ de acuerdo & $74 \%$ de acuerdo & $62 \%$ de acuerdo \\
\hline
\end{tabular}

La Tabla 4 muestra los resultados de las prácticas educativas parentales por conglomerado. Como primer punto se abordó el nivel de exigencia sobre horarios para realizar tareas, ver televisión y videojuegos, uso de computadora y horarios para acostarse.

Dichas preguntas fueron dicotómicas con opciones de respuesta de Si o No. En cuanto a horarios para realizar tareas, los resultados para Metanoiers y Modernos fueron similares. Ambos grupos indicaron en un $86 \%$ y $87 \%$, respectivamente, que si exigen un horario para realizar sus tareas. Por otro lado, los Tradicionales sólo lo exigen en un $68 \%$ de la muestra.

En lo que corresponde a establecer horarios para ver televisión y juegos, $81 \%$ de los Metanoiers respondieron afirmativamente. Los Modernos estuvieron de acuerdo en un 76\%; mientras que los Tradicionales reportaron un 53\%. La misma tendencia se observó en los grupos en cuanto a exigir horarios de uso de computadora se refiere. Siendo así, las respuestas afirmativas fueron de un $78 \%$ para Metanoiers, $59 \%$ para Modernos y $11 \%$ para Tradicionales.

Se consultó a los padres sobre si imponen un horario para acostarse. El 84\% de los Metanoiers respondió afirmativamente, similar al 85\% de los Modernos; mientras que los Tradicionales reportaron un 53\%. En cambio, cuando se consultó sobre la exigencia de un lugar en la casa para la realización de tareas, los Metanoiers respondieron afirmativamente en un 56\%, $87 \%$ los Modernos y $37 \%$ los Tradicionales.

La misma tabla muestra los resultados sobre prácticas educativas relacionadas con la lectura, específicamente sobre leer libros a los hijos o incentivar la lectura en ellos. Los Metanoiers y Modernos presentan porcentajes similares sobre leer libros a sus hijos. Ambos grupos reportan que lo hacen ocasionalmente o muy frecuentemente en un $73 \%$ y $81 \%$, respectivamente.

En contraste, los Tradicionales, en un $63 \%$ reportan que nunca o casi nunca lo hacen. La tendencia se mantiene al consultar sobre el incentivo a la lectura. En este sentido, los Metanoiers 
y Modernos lo hacen ocasionalmente o muy frecuentemente en un $77 \%$ y 93\%, respectivamente; mientras que el $74 \%$ de los Tradicionales, nunca o casi nunca lo hacen.

El $81 \%$ de los Metanoiers y el 70\% de los Modernos, ocasionalmente o muy frecuentemente, discuten con sus hijos sobre temas de actualidad; mientras que el $74 \%$ de los Tradicionales, nunca lo hace. Así mismo, el 53\% de los Metanoiers conversa con sus hijos muy frecuentemente sobre la importancia de la escuela, tal como lo hacen el $81 \%$ de los Modernos; mientras que el $47 \%$ de los Tradicionales nunca lo hace y el 16\% lo hace ocasionalmente.

Entre otras estadísticas, el $48 \%$ de los Metanoiers, ocasionalmente revisa las tareas de sus hijos; mientras que el $86 \%$ de los Modernos lo hace con mucha frecuencia y el $47 \%$ de los Tradicionales nunca lo hace. De igual manera, el 58\% de los Metanoiers, ocasionalmente plantea a sus hijos operaciones matemáticas simples; mientras que los Modernos y Tradicionales lo hacen con mucha frecuencia en un $59 \%$ y $42 \%$, respectivamente.

So consultó a los padres su postura sobre la dificultad de las tareas en la actualidad. Específicamente se pidió que mostraran su acuerdo o desacuerdo con que las tareas de hoy en día son más fáciles que las tareas de antes. Ante esta afirmación, el 56\% de los Metanoiers se mostraron en desacuerdo, los Modernos no presentaron tendencia alguna y los Tradicionales estuvieron de acuerdo en un $62 \%$.

Finalmente, y acorde a lo que se había consultado previamente, se consultó a los padres si disponen de tiempo para ayudar a sus hijos en sus tareas a lo que los tres grupos respondieron afirmativamente en un $75 \%, 74 \%$ y $62 \%$, respectivamente.

Tabla 5. Aspiraciones Parentales

\begin{tabular}{|c|c|c|c|}
\hline Variables & Metanoiers & Modernos & Tradicionales \\
\hline Prepararlo para ENES y universidad & $\begin{array}{l}51 \% \text { muy } \\
\text { importante }\end{array}$ & $\begin{array}{l}71 \% \text { muy } \\
\text { importante }\end{array}$ & $\begin{array}{l}69 \% \text { muy } \\
\text { importante }\end{array}$ \\
\hline $\begin{array}{l}\text { Desarrollar habilidades de } \\
\text { aprendizaje e inteligencia }\end{array}$ & $\begin{array}{l}63 \% \text { muy } \\
\text { importante }\end{array}$ & $\begin{array}{l}71 \% \text { muy } \\
\text { importante }\end{array}$ & $\begin{array}{l}63 \% \text { muy } \\
\text { importante }\end{array}$ \\
\hline $\begin{array}{c}\text { Formarlo como persona autónoma y } \\
\text { responsable }\end{array}$ & $\begin{array}{l}84 \% \text { muy } \\
\text { importante }\end{array}$ & $\begin{array}{l}82 \% \text { muy } \\
\text { importante }\end{array}$ & $\begin{array}{l}56 \% \text { muy } \\
\text { importante }\end{array}$ \\
\hline $\begin{array}{l}\text { Entregarle conocimientos para un } \\
\text { buen trabajo }\end{array}$ & $\begin{array}{l}83 \% \text { muy } \\
\text { importante }\end{array}$ & $\begin{array}{l}84 \% \text { muy } \\
\text { importante }\end{array}$ & $\begin{array}{l}54 \% \text { muy } \\
\text { importante }\end{array}$ \\
\hline
\end{tabular}

Como última dimensión de las estrategias educativas familiares, se realizaron preguntas sobre las aspiraciones parentales. La Tabla 5 muestra los resultados encontrados por segmento. Esta dimensión fue medida a través de una escala de likert de 4 niveles que iba desde nada importante hasta muy importante. 
En lo que respecta a preparar a los estudiantes para el Examen Nacional para la Educación Superior - ENES, los Metanoiers indicaron en un 51\% que era muy importante, mientras que los Modernos lo reportaron en un 71\% y los Tradicionales en un 69\%. Así mismo, en los resultados frente a la consulta de desarrollar habilidades de aprendizaje e inteligencia, los Metanoiers y los Tradicionales, señalaron en un 63\% que era muy importante, mientras que los Modernos lo hicieron en un $71 \%$.

Formar a los hijos como personas autónomas y responsables, como una variable de las aspiraciones parentales, se mostró como un aspecto muy importante por el $84 \%$ de los Metanoiers, el $82 \%$ de los Modernos y el 56\% de los Tradicionales. Finalmente, la variable de entregar conocimientos para la obtención de un buen trabajo, se mostró como muy importante para el $83 \%$ de los Metanoiers, el 84\% de los Modernos y el 54\% de los Tradicionales.

\section{Conclusiones}

Una vez analizados los resultados del estudio se pudo determinar que los padres tienen diferentes prácticas en lo que respecta a la educación de sus hijos. Estas diferencias permitieron agrupar a los integrantes de la muestra en tres segmentos denominados Metanoiers, Modernos y Tradicionales. Estos tres grupos presentan diferencias en las cuatro dimensiones de las EEF.

Los Metanoiers, en promedio, tienen entre 30 y 40 años de edad; poseen educación universitaria completa o superior; tienen trabajo o negocio permanente; suelen tener entre 1 y 2 hijos menores de edad; poseen ingresos superiores a $\$ 1,500$; no acostumbran leer libros con mucha frecuencia; no suelen practicar juegos didácticos con sus hijos; y confían en la información de internet para ayudar a la resolución de tareas y dudas de los estudiantes.

En referencia a la disposición parental hacia la escuela, los Metanoiers, en su mayoría, casi siempre asisten a las reuniones de apoderados; no son muy comprometidos con el voluntariado para actividades escolares, ni en el acompañamiento al profesor en actividades fuera de la escuela. La razón de la falta de involucramiento es el trabajo.

Este mismo grupo, suele presentar las siguientes prácticas educativas parentales: exigen un horario para realizar las tareas, establecen horarios para la televisión y juegos, establecen horarios para el uso de computadora, imponen hora de acostarse, no son exigentes con respecto al lugar donde se desarrollan las tareas, con mucha frecuencia leen libros a sus hijos, les cuentan historias y cuentos inventados, e incentivan la lectura por cuenta propia.

Entre otras prácticas, discuten con los niños sobre temas de actualidad, conversan con ellos sobre la importancia de la escuela, no acostumbran revisarles las tareas escolares ni plantearles operaciones matemáticas básicas. Quienes conforman este grupo consideran que las tareas de la actualidad son más difíciles que las tareas de antes y disponen de tiempo para ayudar a sus hijos en dichas tareas.

Por último, los Metanoiers presentan las siguientes aspiraciones parentales con respecto a la escuela: que formen a sus hijos como personas autónomas y responsables, y que les entreguen 
los conocimientos necesarios para que obtengan un buen trabajo. En un menor grado de importancia indicaron que esperan que los preparen para el ENES y la universidad, así como que desarrollen sus habilidades de aprendizaje e inteligencia.

En referencia al grupo de los Modernos, en promedio, se encuentran entre los 25 y 35 años de edad; poseen educación básica completa o media completa; se dedican principalmente a labores domésticas y trabajos eventuales; tienen entre 2 y 3 hijos menores de edad; poseen ingresos de hasta $\$ 470$; acostumbran leer libros al menos una vez por semana; suelen practicar juegos didácticos con sus hijos; y confían en familiares al momento de resolver tareas y dudas de los estudiantes.

La disposición de los Modernos hacia la escuela suele caracterizarse por siempre asistir a las reuniones de apoderados, casi nunca participar de voluntariado en actividades dentro de la escuela y nunca acompañar a los profesores en actividades fuera de la escuela. La falta de voluntariado y acompañamiento se justifica por trabajo o tareas del hogar.

Entre las prácticas educativas que tienen los Modernos se incluyen: la exigencia de un horario para realizar tareas, el establecimiento de horarios para televisión y juegos, la imposición de un horario para acostarse, y la exigencia de un lugar específico para realizar tareas. Entre otras características, no son restrictivos con respecto a horarios para el uso de computadoras, con mucha frecuencia leen libros a sus hijos e incentivan en ellos la lectura, a menudo optan por contar a sus hijos historias y cuentos inventados, discuten con ellos sobre temas de actualidad, les conversan sobre la importancia de la escuela, revisan sus tareas escolares, en ocasiones les plantean operaciones matemáticas básicas; y en general, disponen de tiempo para ayudarlos en sus tareas.

Los Modernos, entre sus aspiraciones con respecto a la escuela, esperan que ésta los prepare para el ENES y la universidad, les ayude a desarrollar habilidades de aprendizaje e inteligencia; y con mayor relevancia, los formen como personas autónomas y responsables, al mismo tiempo que les entreguen conocimientos necesarios para que lleguen a obtener un buen trabajo.

Por otra parte, el grupo de los Tradicionales, en promedio, tienen más de 40 años; poseen educación básica completa o inferior; se dedican a tareas del hogar o trabajos eventuales; tienen entre 1 y 2 hijos menores de edad; ganan menos de \$366 mensuales; en su mayoría leen libros al menos una vez por semana; nunca practican juegos didácticos con sus hijos y confían en sus familiares para cuando necesitan ayuda en resolución de tareas o dudas de los estudiantes. Siempre asisten a las reuniones de apoderados y nunca realizan voluntariado o ayuda a los profesores, principalmente por ocupaciones del hogar o trabajo.

Este grupo de apoderados no suele ser muy exigente en el horario para realizar tareas, no exige horarios para ver televisión o jugar, no establece horarios para uso de computadoras, no impone hora de acostarse y no exige realizar tareas en lugares específicos. No acostumbran leerles libros a sus hijos ni incentivar la lectura en ellos, nunca les cuentan historias inventadas ni hablan con ellos sobre temas de actualidad, tampoco conversan con sus hijos sobre la importancia de la escuela ni revisan sus tareas escolares. Con mucha frecuencia les plantean 
operaciones matemáticas básicas. Consideran que las tareas de hoy en día son más fáciles que las de antes y reportan que si tienen tiempo para revisar las tareas de sus hijos.

En cuanto a las aspiraciones parentales, los Tradicionales consideran que lo principal es prepararlos para el ENES y la universidad. En segundo lugar, desarrollar sus habilidades de aprendizaje e inteligencia. No señalan fuertemente, como aspiración de la escuela, que los formen como personas autónomas y responsables, ni que les entreguen conocimientos para la obtención de un buen trabajo.

En conclusión, las diferentes dimensiones de las EEF no son iguales para todos los apoderados. El estudio identifica tres segmentos a través de la técnica de análisis de conglomerados, no obstante esta técnica no es inferencial sino descriptiva, por lo que no se puede generalizar para toda la población de Guayaquil. En este sentido, se recomienda que se replique el estudio en otros lugares o muestras distintas para poder realizar una comparación de resultados.

\section{Bibliografía}

Bernard Van Leer Foundation. (2009). La Primera Infancia en Perspectiva: Programas eficace para la primera infancia. Obtenido de The Open University: https://bernardvanleer.org/app/uploads/2016/03/Programas_eficaces_para_la_primera_inf ancia2ba1.pdf

Blanco, R. (2012). Una atención y educación de calidad en la primera infancia puede hacer la diferencia. Docencia No. $48,6$.

Bourdieu, P., \& Passeron, J. C. (1977). La Reproducción: Elementos para una teoría de enseñanza. Barcelona: Laia.

Bueno, E. (1994). Fundamentos de Economía y Organización Industrial. Mc Graw Hill.

Campos, A. L. (2010). La Neuroeducación: una nueva aliada para los programas de atención y educación de la primera infancia. En A. L. Campos, Primera Infancia: Una mirada desde la Neuroeducación (págs. 11-18). Lima: Cerebrum: Centro Iberoamericano de Neurociencias, Educación y Desarrollo Humano.

CDE. (2010). Departamento de Educación de California. Obtenido de Guias para programas de aprendizaje y desarrollo infantil: http://www.cde.ca.gov/sp/cd/re/documents/itproggdlnesspan.pdf

Contreras, D., Figueroa, J., \& Espina, M. (2012). FAMILIA-ESCUELA Trabajando Juntos. Santiago: Fondo de las Naciones Unidas para la Infancia y Fundación Collahuasi.

DABS. (2006). Folleto Documento Pautas y Prácticas de Crianza. Obtenido de Departamento Administrativo de Bienestar Social del distrito de Bogotá: file://C:/Users/sybaq_000/Downloads/Folleto-Pautas-practicas-crianza2\%20(1).pdf 
De-La-Fuente, S. (2011). Análisis Conglomerados. Facultad de Ciencias Económicas y Empresariales. Universidad Autónoma de Madrid .

Early Childhood Learning \& Knowledge Center. (15 de Agosto de 2011). Marco de participación en Head Start de los padres, la familia y la comunidad (PFCE). Obtenido de Se promueve la participación familiar y la preparación para la escuela desde la etapa prenatal hasta la edad de 8 años: http://eclkc.ohs.acf.hhs.gov/hslc/Espanol/participacion/docs/pfceframework-spanish.pdf

Epstein, J. (2013). Programas efectivos de Involucramiento familiar en las escuelas: estudios y prácticas. Santiago: Fundación CAP.

García, F. (29 y 30 de Noviembre de 2001). Modelo Ecológico / Modelo Integral de Intervención en atención Temprana. Madrid, España.

Gubbins, V. (2014). Estrategias educativas de familias de clase alta: un estudio exploratorio. Psyche. Versión online, 1-17.

Gubbins, V. (2012). Familia y escuela: tensiones, reflexiones y propuestas. Docencia No. 46 , 64 -73 .

Gubbins, V., \& Ibarra, S. (Mayo de 2016). Estrategias Educativas Familiares en Enseñanza Básica: Análisis Psicométrico de una Escala de Prácticas Parentales. Psykhe vol. 25 no.1 , 1-17.

Hoel, P., \& Jessen, R. (1983). EStadística Básica para Negocios y Economía. CECSA.

Izaguirre, J., \& Medina, V. (2017). Análisis de Determinantes de las Prácticas Educativas Parentales en Hogares con Hijos entre Primero y Cuarto Año de Educación Básica de la Ciudad de Guayaquil. INNOVA Research Journal 2017, Vol 2, No. 8, 104 - 118.

López, M. E., \& Caspe, M. (Junio de 2014). Involucramiento Familiar para el aprendizaje en cualquier momento y lugar. Obtenido de Harvard Family Research Project: http://www.hfrp.org/

Merizalde, J. (2017). Estudio descriptivo sobre las prácticas parentales asociadas al aprendizaje escolar de padres de familia de estudiantes de lero a 4to grado de educación básica de unidades educativas del cantón Guayaquil. Guayaquil: Universidad Casa Grande.

Ministerio de Educación, C. y. (16-18 de Abril de 2015). Las relaciones entre familia y escuela. Experiencias y buenas prácticas. Documento base. Obtenido de XXIII Encuentro de Consejos Escolares Autonómicos y del Estado: http://www.mecd.gob.es/dctm/cee/encuentros/23encuentro/23encuentroceaedocumentoba se2015.pdf?documentId=0901e72b81cba426

Rivera, M., \& Milicic, N. (2006). Alianza Familia-Escuela: Percepciones, Creencias, Expectativas y Aspiraciones de Padres y Profesores de Enseñanza General Básica. Psykhe , 1-14.

Sallés, C., \& Ger, S. (2011). Las competencias parentales en la familia contemporánea: 
descripción, promoción y evaluación. Educación Social: Revista de Intervención Socioeducativa , 25-47.

Solís, D. (2013). El Buen Vivir empieza en la Infancia. MIESpacio - Revista del Ministerio de Inclusión Económica y Social, 3.

UNICEF. (Septiembre de 2014). La niñez y la adolescencia en el Ecuador contemporáneo: avances y brechas en el ejercicio de derechos. Obtenido de Observatorio Social del Ecuador:

http://www.unicef.org/ecuador/NA_EcuadorContemporaneo_ultima_version.pdf

Weiss, H., Bouffard, S., Bridgall, B., \& Edmund, G. (Diciembre de 2009). Hacia un nuevo enfoque sobre el involucramiento de la familia en la educación: apoyar a las familias para promover la equidad en la educación. Obtenido de Harvard Family Research: http://www.hfrp.org/publications-resources/browse-our-publications/reframing-familyinvolvement-in-education-supporting-families-to-support-educational-equity

Ycaza, S. (2017). Estudio descriptivo y correlacional sobre las Estrategias Educativas Familiares de padres de familia de estudiantes de segundo año de educación básica de unidades educativas del cantón Guayaquil. Guayaquil: Universidad Casa Grande. 